\title{
Local spin polarization in high energy heavy ion collisions
}

\author{
Hong-Zhong Wu ${ }^{1,},{ }^{*}$ Long-Gang Pang $\oplus^{2, \dagger}$ Xu-Guang Huang, ${ }^{3,4, \ddagger}$ and Qun Wang ${ }^{1, \S}$ \\ ${ }^{1}$ Department of Modern Physics, University of Science and Technology of China, Hefei, Anhui 230026, China \\ ${ }^{2}$ Nuclear Science Division, MS 70R0319, Lawrence Berkeley National Laboratory, Berkeley, California 94720, USA \\ ${ }^{3}$ Physics Department and Center for Particle Physics and Field Theory, Fudan University, Shanghai 200433, China \\ ${ }^{4}$ Key Laboratory of Nuclear Physics and Ion-beam Application (MOE), Fudan University, Shanghai 200433, China
}

(Received 11 July 2019; revised manuscript received 7 September 2019; published 29 October 2019)

\begin{abstract}
We reexamine the azimuthal angle dependence of the local spin polarization of hyperons in heavy ion collisions at $200 \mathrm{GeV}$ in the framework of the $(3+1)$-dimensional viscous hydrodynamic model CLVisc. Two different initial conditions are considered in our simulation: the optical Glauber initial condition without an initial orbital angular momentum and the AMPT initial condition with an initial orbital angular momentum. We find that the azimuthal angle dependence of the hyperon polarization strongly depends on the choice of the so-called spin chemical potential $\Omega_{\mu \nu}$. With $\Omega_{\mu \nu}$ chosen to be proportional to the temperature vorticity, our simulation shows qualitatively coincidental results with the recent measurements at the BNL Relativistic Heavy Ion Collider for both the longitudinal and the transverse polarization. We argue that such a coincidence may be related to the fact that the temperature vorticity is approximately conserved in the hot quark-gluon matter.
\end{abstract}

DOI: 10.1103/PhysRevResearch.1.033058

\section{INTRODUCTION}

It is well known that the rotation and the spin polarization are correlated and can be converted to each other in materials $[1,2]$. Recently, the polarization of electrons in a vortical fluid has been observed [3]. Similar phenomena also exist in high energy heavy ion collisions in which huge orbital angular momenta (OAM) are produced in peripheral collisions [4-8] (for a recent review, see, e.g., Ref. [8]). The huge OAM are distributed into the quark-gluon plasma created in heavy ion collisions in the form of local vorticity [9-12], which results in the local polarization of hadrons along the vorticity direction $[13,14]$ due to the spin-orbit coupling $[4,6]$. The net effect of the local polarization at all space-time points on the freeze-out hypersurface gives the global polarization in the direction of the reaction plane or the OAM of two colliding nuclei [4-6,8,15-17].

The global polarization of $\Lambda$ and $\bar{\Lambda}$ has been measured by the STAR Collaboration in $\mathrm{Au}+\mathrm{Au}$ collisions at $\sqrt{s_{N N}}=$ 7.7-200 GeV [18,19]. The data show a decreasing trend in collision energies from about $2 \%$ at $7.7 \mathrm{GeV}$ to about $0.3 \%$ at $200 \mathrm{GeV}$.

There are several theoretical approaches which have been developed to study the global and local polarization in heavy

\footnotetext{
*whz168@mail.ustc.edu.cn

†lgpang@1bl.gov

*huangxuguang@fudan.edu.cn

§qunwang@ustc.edu.cn
}

Published by the American Physical Society under the terms of the Creative Commons Attribution 4.0 International license. Further distribution of this work must maintain attribution to the author(s) and the published article's title, journal citation, and DOI. ion collisions. The statistic-hydro model is based on the spinvorticity coupling in the thermal distribution function $[13,20$ $23]$. So the average spin polarization is proportional to the socalled thermal vorticity (see the definition in the next section) if the thermal vorticity is small. Another theoretical approach is the Wigner function (WF) formalism [24-30], which has been revived [31-39] to study the chiral magnetic effect [40-42] (for reviews, see, e.g., Refs. [43-46]) and the chiral vortical effect [31,47-51] for massless fermions. Recently, the kinetic theory for spin-1/2 massive fermions has been formulated in the WF framework [14,52-55], which is useful in describing the evolution of the spin polarization. This is because the axial vector component gives the spin phase-space distribution of fermions. At equilibrium, when the thermal vorticity is small, the spin polarization of fermions from the WF formalism is proportional to the thermal vorticity, consistent with the statistic-hydro model.

To describe the STAR data on the global polarization of hyperons which is along the direction of the reaction plane, the hydrodynamic and transport models have been used to calculate the vorticity field [11,12,56-62]. In the hydrodynamic framework, the velocity and in turn the vorticity fields in the fireball can be obtained naturally. The transport models describe the phase-space evolution of a particle system through collisions among particles, so the position and momentum of each particle in the system at any time is given. To obtain the fluid velocity and then the vorticity at one space-time point, a suitable coarse graining procedure has to be used. Once the vorticity field is obtained, the global polarization of hyperons can be calculated from an integral over the freeze-out hypersurface which agrees well with the data [61-65].

The polarization of hyperons as a function of the azimuthal angle in the transverse plane has been recently measured in the STAR experiment $[19,66]$. However the data for the 
polarization along both the longitudinal and the transverse directions cannot be described by the hydrodynamic models [including a multiphase transport (AMPT) model from which the vorticity field is extracted by the coarse graining method] $[62,67,68]$ based on the coupling of the thermal vorticity and the spin at equilibrium. There is a sign difference between the data and these model calculations. Although one model based on the chiral kinetic theory can explain the sign of the data [69], it cannot reproduce the magnitude of the data. Recent studies have shown that the feed-down effects cannot resolve the sign difference [70,71].

The disagreement between theories and experiments indicates that the spin degree of freedom may not reach equilibrium in the fireball and thus the spin polarization may not be determined by the thermal vorticity. The relation between spin and thermal vorticity is dictated by the condition of local thermodynamic equilibrium if the spin tensor does not play a physical role [72]. This calls for new approaches; for example, the spin can be treated as an independent dynamical variable in the spin kinetic theory and spin hydrodynamics, or dissipative terms should be considered which are possibly larger than believed. Recently, the framework of spin hydrodynamics was developed [73-75]. The spin evolution based on particle collisions was derived [76]. The purpose of the present paper is not to make a numerical study based on these new approaches; instead, our purpose is not that ambitious: we explore different choices of the so-called "spin chemical potential" $\Omega_{\mu \nu}$ and calculate the corresponding local hyperon polarization. The underlying reason is that, beyond global equilibrium, the thermal vorticity is not guaranteed to be the spin chemical potential, and thus the latter becomes a free parameter $[72,74,75]$. In the $(3+1)$-dimensional $[(3+1) \mathrm{D}]$ hydrodynamic model CLVisc [77,78], we assume that the spin chemical potential $\Omega_{\mu \nu}$ is still determined by the fluid velocity and temperature (or equivalently the energy density). This means that $\Omega_{\mu \nu}$, being an antisymmetric tensor, can be regarded as a type of vorticity (with appropriate normalization to make the dimension correct). We thus explore four different definitions for $\Omega_{\mu \nu}$ or vorticity and calculate the local hyperon polarization and compare with the data. In our hydrodynamic simulation, we examine two different initial conditions: the optical Glauber initial condition without an initial OAM and the AMPT initial condition with an initial OAM.

The paper is organized as follows. In Sec. II we give a brief discussion about our motivation. In Sec. III we introduce our hydrodynamic model which we use for the simulation. We present our numerical results in Sec. IV. We give some discussions in Sec. V. Finally, we give a summary of our results in Sec. VI.

\section{SPIN POLARIZATION AND VORTICITY}

The thermodynamic equilibrium in quantum field theory can be described by the density operator $\hat{\rho}$. Its form at local equilibrium can be obtained by maximizing the entropy $S=$ $-\operatorname{Tr}(\hat{\rho} \ln \hat{\rho})$ with fixed densities of the energy momentum, the angular momentum, and the conserved charge current on a spacelike hypersurface $\Sigma^{\mu}=n^{\mu} \Sigma$ pointing to a timelike direction $n^{\mu}$ [79-82],

$$
\begin{aligned}
n_{\mu} \operatorname{Tr}\left(\hat{\rho} \hat{T}^{\mu \nu}\right) & =n_{\mu} T^{\mu \nu}, \\
n_{\mu} \operatorname{Tr}\left(\hat{\rho} \hat{J}^{\mu, \alpha \beta}\right) & =n_{\mu} J^{\mu, \alpha \beta}, \\
n_{\mu} \operatorname{Tr}\left(\hat{\rho} \hat{N}^{\mu}\right) & =n_{\mu} N^{\mu},
\end{aligned}
$$

where $\hat{T}^{\mu \nu}, \hat{J}^{\mu, \alpha \beta}$, and $\hat{N}^{\mu}$ are the density operators of the energy-momentum tensor, the angular-momentum tensor, and the conserved charge current, respectively. Note that $\hat{T}^{\mu \nu}$ is not necessarily symmetric. The quantities $T^{\mu \nu}, J^{\mu, \alpha \beta}$, and $N^{\mu}$ are their expectation values. For simplicity we call $\hat{T}^{\mu \nu}$ $\left(T^{\mu \nu}\right)$ and $\hat{J}^{\mu, \alpha \beta}\left(J^{\mu, \alpha \beta}\right)$ the energy-momentum tensor and angular-momentum tensor, respectively, though they are actually tensor densities. The angular-momentum density operator includes the orbital and spin parts:

$$
\hat{J}^{\mu, \alpha \beta}=x^{\alpha} \hat{T}^{\mu \beta}-x^{\beta} \hat{T}^{\mu \alpha}+\hat{S}^{\mu, \alpha \beta} .
$$

Thus, the second constraint in Eq. (1) can be equivalently expressed as

$$
n_{\mu} \operatorname{Tr}\left(\hat{\rho} \hat{S}^{\mu, \alpha \beta}\right)=n_{\mu} S^{\mu, \alpha \beta} .
$$

The form of the density operator under the constraints (1), or with the second constraint in Eq. (1) being replaced by the constraint (3), that maximizes the entropy reads

$\hat{\rho}_{\mathrm{LE}}=\frac{1}{Z_{\mathrm{LE}}} \exp \left[-\int d \Sigma_{\mu}\left(\hat{T}^{\mu v} \beta_{\nu}-\frac{1}{2} \Omega_{\alpha \beta} \hat{S}^{\mu, \alpha \beta}-\zeta \hat{N}^{\mu}\right)\right]$,

where $\beta_{\nu}, \Omega_{\alpha \beta}$, and $\zeta$ are Lagrangian multipliers which have physical meanings: $\beta_{v}=u_{v} / T$ with $u_{\nu}$ being the four-velocity and $T$ being the temperature, $\zeta=\mu / T$ with $\mu$ being the chemical potential, and $\Omega_{\alpha \beta}$ plays the role of the chemical potential for the angular momentum. ${ }^{1}$ In the following, we simply call $\Omega_{\alpha \beta}$ the spin chemical potential as it determines the spin polarization at local equilibrium. The density operator $\hat{\rho}_{\mathrm{LE}}$ in Eq. (4) defines the local thermal equilibrium and in general depends on the time.

In relativistic hydrodynamics, in order to obtain the spin vector, we need to first obtain $T, u^{\mu}$, and $\Omega_{\mu \nu}$ by solving the hydrodynamic equations in which the spin degree of freedom (or equivalently $\Omega_{\mu v}$ ) is treated on the same footing as $T$ and $u^{\mu}$. Such a framework is the spin hydrodynamics $[73,75]$. However, the numerical spin hydrodynamics has not been established yet. Therefore we adopt an usual $(3+1) \mathrm{D}$ hydrodynamic model, CLVisc [77,78], which can give the space-time evolution of $T$ and $u^{\mu}$. Since $\Omega_{\mu \nu}$ is antisymmetric, we then assume that $\Omega_{\mu \nu}$ can be constructed from $T$ and $u^{\mu}$ as $\Omega_{\mu \nu}=-(1 / 2) \lambda(T)\left[\partial_{\mu}\left(g(T) u_{v}\right)-\partial_{v}\left(g(T) u_{\mu}\right)\right] \equiv$ $\lambda(T) \omega_{\mu \nu}$ or its projections, where $\lambda$ and $g$ are scalar functions of $T$ and $\omega_{\mu \nu}$ is the vorticity tensor. ${ }^{2}$ In our numerical

\footnotetext{
${ }^{1}$ More precisely, it is $T \Omega_{\alpha \beta}$ that plays the role of a chemical potential for the angular momentum.

${ }^{2}$ In principle, it is also allowed to use the Hodge dual of the vorticity tensor to construct $\Omega_{\mu \nu}$. However, when the global equilibrium is approached, it is known that $\Omega_{\mu \nu}$ should approach the thermal vorticity up to a constant (depending on the symmetry properties of $\left.T^{\mu \nu}\right)$. We therefore do not consider such a possibility here.
} 
simulation, four types of vorticity are considered, namely, the kinematic vorticity, the relativistic extension of the nonrelativistic vorticity (NR vorticity), the thermal vorticity, and the temperature vorticity ( $\mathrm{T}$ vorticity). All these four types of vorticities have definite physical meaning and have been widely studied for quite some time in the literature. For example, in Ref. [9] the properties of the kinematic, thermal, and $\mathrm{T}$ vorticities have been studied in heavy ion collisions, and in Ref. [83] the NR vorticity is defined in a study of $\Lambda$ polarization in heavy ion collisions.

The kinematic vorticity is defined by

$$
\omega_{\mu \nu}^{(K)}=-\frac{1}{2}\left(\partial_{\mu} u_{\nu}-\partial_{\nu} u_{\mu}\right)
$$

where $u^{\mu}=\gamma(1, v)$ is the four-velocity and $\gamma$ is the Lorentz factor. The mass dimension of the kinematic vorticity is 1 . The kinematic vorticity tensor can be decomposed into the part parallel and the part orthogonal to the fluid velocity:

$$
\omega_{\mu \nu}^{(K)}=\varepsilon_{v} u_{\mu}-\varepsilon_{\mu} u_{v}+\epsilon_{\nu \mu \rho \eta} u^{\rho} \omega^{\eta},
$$

where $\varepsilon_{\mu}=-(1 / 2) u^{\nu} \partial_{\nu} u_{\mu}$ and $\omega^{\mu}=(1 / 2) \epsilon^{\mu \nu \rho \sigma} u_{\nu}\left(\partial_{\rho} u_{\sigma}\right)$. In comparison with the decomposition of the electromagnetic field strength $F^{\mu \nu}$, the vector $\varepsilon^{\mu}$ is like an "electric" field while the vorticity vector $\omega^{\mu}$ is like a "magnetic" field. It is clear that $\omega^{\mu}$ is a direct extension of the vorticity defined in nonrelativistic hydrodynamics, $\omega=(1 / 2) \nabla \times \boldsymbol{v}$. We thus define the last term in Eq. (6) as the NR-vorticity tensor [83]:

$$
\omega_{\mu \nu}^{(\mathrm{NR})}=\epsilon_{\nu \mu \rho \eta} u^{\rho} \omega^{\eta} .
$$

Similar to $\omega_{\mu \nu}^{(\mathrm{NR})}$, there has been an attempt to use the spatial components of the thermal vorticity as the spin chemical potential to study the longitudinal spin polarization of hyperons [84].

The $\mathrm{T}$ vorticity is defined by

$$
\begin{aligned}
\omega_{\mu \nu}^{(T)} & =-\frac{1}{2}\left[\partial_{\mu}\left(T u_{\nu}\right)-\partial_{\nu}\left(T u_{\mu}\right)\right] \\
& =T \omega_{\mu \nu}^{(K)}+\frac{1}{2}\left(u_{\mu} \partial_{\nu} T-u_{\nu} \partial_{\mu} T\right) \\
& \equiv T \omega_{\mu \nu}^{(K)}+\omega_{\mu \nu}^{(T)}(T),
\end{aligned}
$$

where the temperature enters the space-time derivative. The mass dimension of the temperature vorticity is 2 . We have decomposed $\omega_{\mu \nu}^{(T)}$ into the part involving the space-time gradient of the temperature and the part without it, which is proportional to the kinematic vorticity.

An important property of the $\mathrm{T}$ vorticity is that it obeys a conservation law $[9,11,85]$. Suppose $\Xi$ is a two-dimensional hypersurface and $C$ is its boundary, thus the flux of the temperature vorticity on $\Xi$ is equal to the corresponding circulation of $T u^{\mu}$ along the boundary $C$ :

$$
\int_{\Xi} \omega_{\mu \nu}^{(T)} d x^{\mu} \wedge d x^{\nu}=-\oint_{C} T u_{\mu} d x^{\mu} .
$$

Since the viscosity of the hot matter in the fireball is small, we can approximately apply the Euler equation for an ideal fluid,

$$
(\varepsilon+P) \frac{d}{d \tau} u^{\mu}=\nabla^{\mu} P,
$$

where $\varepsilon$ and $P$ are the energy density and pressure, respectively, $d / d \tau=u^{\mu} \partial_{\mu}$ is the comoving time derivative, and $\nabla_{\mu}=\partial_{\mu}-u_{\mu}(d / d \tau)$. Rewriting the Euler equation in the following form,

$$
\frac{d}{d \tau}\left(T u^{\mu}\right)=\partial^{\mu} T
$$

one easily finds

$$
\frac{d}{d \tau} \oint T u_{\mu} d x^{\mu}=\oint \partial_{\mu} T d x^{\mu}=0 .
$$

This is the relativistic Helmholtz-Kelvin theorem: the flux of the T-vorticity tensor is conserved with the fluid cell along $u^{\mu}$. We will see that this imposes a strong influence on the spin polarization.

The thermal vorticity $\omega_{\mu \nu}^{(\text {th) }}$ is defined by

$$
\begin{aligned}
\omega_{\mu \nu}^{(\mathrm{th})} & =-\frac{1}{2}\left[\partial_{\mu}\left(\beta u_{\nu}\right)-\partial_{\nu}\left(\beta u_{\mu}\right)\right] \\
& =\frac{1}{T} \omega_{\mu \nu}^{(K)}-\frac{1}{2 T^{2}}\left(u_{\mu} \partial_{\nu} T-u_{\nu} \partial_{\mu} T\right) \\
& =\frac{1}{T} \omega_{\mu \nu}^{(K)}+\omega_{\mu \nu}^{(\mathrm{th})}(T),
\end{aligned}
$$

where $\beta=1 / T$. The thermal vorticity is dimensionless. Similar to $\omega_{\mu \nu}^{(T)}$ we have also decomposed $\omega_{\mu \nu}^{(\text {th })}$ into the part involving the space-time gradient of the temperature and the part without it, which is proportional to $\omega_{\mu \nu}^{(K)}$. We see in Eqs. (8) and (13) that $\omega_{\mu \nu}^{(T)}(T)$ and $\omega_{\mu \nu}^{(\mathrm{th})}(T)$ have the opposite sign.

The importance of the thermal vorticity relies on the fact that at global equilibrium, $\Omega_{\mu \nu}$ equals to $\omega_{\mu \nu}^{(\text {th })}$ provided that the energy-momentum tensor $\hat{T}^{\mu \nu}$ has a nonvanishing antisymmetric component $[72,75,86]$. This can be seen from the following procedure (the analysis based on the dissipative spin hydrodynamics or the kinetic theory gives the same conclusion). The global equilibrium is the state in which the density operator (4) becomes independent of the choice of the hypersurface $\Sigma_{\mu}$, so that

$$
\hat{T}^{\mu \nu} \partial_{\mu} \beta_{\nu}-\frac{1}{2} \hat{S}^{\mu, \alpha \beta} \partial_{\mu} \Omega_{\alpha \beta}+\frac{1}{2}\left(\hat{T}^{\alpha \beta}-\hat{T}^{\beta \alpha}\right) \Omega_{\alpha \beta}=0,
$$

where we used $\partial_{\mu} \hat{S}^{\mu, \alpha \beta}=\hat{T}^{\beta \alpha}-\hat{T}^{\alpha \beta}$. The above condition is fulfilled when ${ }^{3}$

$$
\begin{aligned}
\partial_{\mu} \beta_{v}+\partial_{\nu} \beta_{\mu} & =0, \\
\partial_{\mu} \Omega_{\alpha \beta} & =0, \\
\Omega_{\alpha \beta} & =\omega_{\alpha \beta}^{(\text {th })} .
\end{aligned}
$$

Note that if $\hat{T}^{\mu \nu}$ is symmetric, the spin tensor is conserved $\partial_{\mu} \hat{S}^{\mu, \alpha \beta}=0$ and the third condition in Eq. (15) does not hold, which means that $\Omega_{\mu \nu}$ remains an independent variable even at global equilibrium. Note that the first line of Eq. (15) is called the Killing equation [87], whose solution is $\beta_{\mu}=b_{\mu}+$ $\omega_{\mu \alpha}^{(\text {th) }} x^{\alpha}$, where $b_{\mu}$ and $\omega_{\mu \alpha}^{(\text {th) }}$ are constants.

\footnotetext{
${ }^{3} \mathrm{We}$ also note that these are sufficient but not necessary conditions for global equilibrium. For example, for conformal fluid, the righthand side of the first condition can be relaxed to $\phi(x) g_{\mu \nu}$, with $\phi$ being a scalar [39].
} 

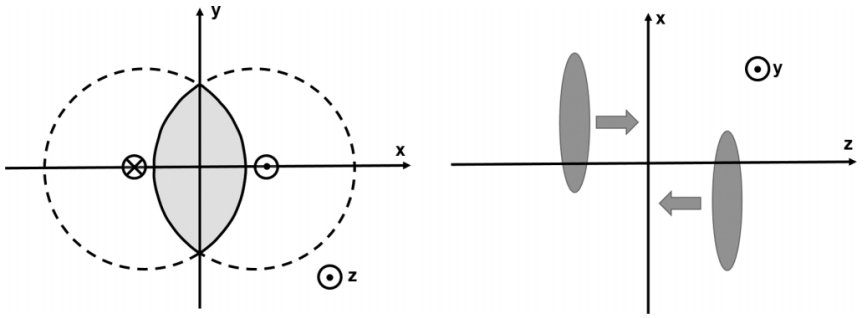

FIG. 1. The coordinate system in the transverse plane. The initial $\mathrm{OAM}$ is along the $-y$ direction.

For spin-1/2 fermions at local equilibrium, when $\omega_{\mu \nu}^{(\text {th) }}$ is small, the average spin vector (defined as the PauliLubanski vector) over the hypersurface $\Sigma_{\mu}$ can be expressed as $[13,14,86]$

$$
\begin{aligned}
S^{\mu}(p)= & -\frac{1}{8 m} \epsilon^{\mu \rho \sigma \tau} p_{\tau} \frac{\int d \Sigma_{\lambda} p^{\lambda} \omega_{\rho \sigma}^{(\mathrm{th})} f_{\mathrm{FD}}\left(1-f_{\mathrm{FD}}\right)}{\int d \Sigma_{\lambda} p^{\lambda} f_{\mathrm{FD}}} \\
& +O\left[\left(\omega_{\mu \nu}^{\text {(th) }}\right)^{2}\right],
\end{aligned}
$$

where $f_{\mathrm{FD}}=1 /\left[\exp \left(p_{\mu} \beta^{\mu}-\zeta\right)+1\right]$ is the Fermi-Dirac distribution, normally $\Sigma_{\mu}$ is chosen as the freeze-out hypersurface for hyperon polarization at the freeze-out. In the calcula-
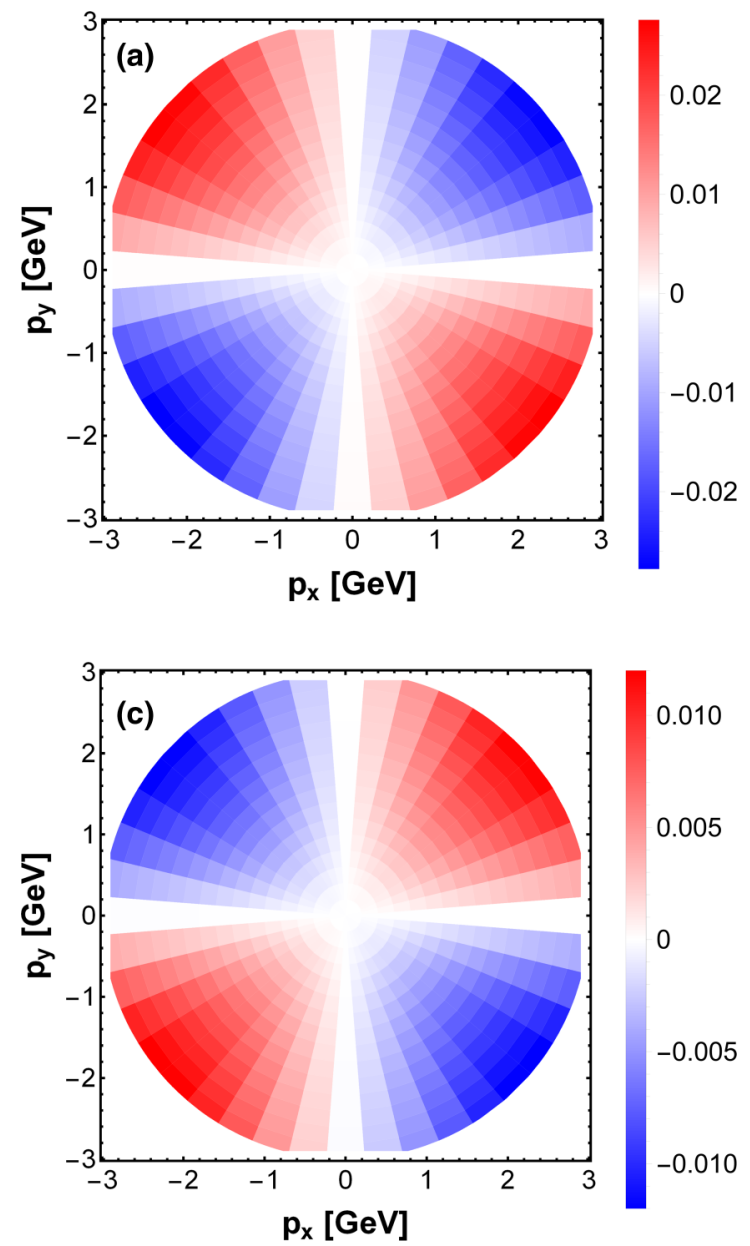

tion we set $\zeta=0$ as the net baryon density is almost zero in the hot fireball created in heavy ion collisions at high energies. In this paper, we assume that Eq. (16) can be generalized by replacing $\omega_{\mu \nu}^{(\mathrm{th})}$ with the spin chemical potential $\Omega_{\mu \nu}$ as

$$
\begin{aligned}
S^{\mu}(p)= & -\frac{1}{8 m} \epsilon^{\mu \rho \sigma \tau} p_{\tau} \frac{\int d \Sigma_{\lambda} p^{\lambda} \Omega_{\rho \sigma} f_{\mathrm{FD}}\left(1-f_{\mathrm{FD}}\right)}{\int d \Sigma_{\lambda} p^{\lambda} f_{\mathrm{FD}}} \\
& +O\left(\Omega_{\mu \nu}^{2}\right),
\end{aligned}
$$

where we consider four types of vorticities as the spin chemical potentials, namely, $\Omega_{\rho \sigma}=\frac{1}{T} \omega_{\rho \sigma}^{(K)}, \frac{1}{T^{2}} \omega_{\rho \sigma}^{(T)}, \omega_{\rho \sigma}^{(\text {th })}$, and $\frac{1}{T} \omega_{\rho \sigma}^{(\mathrm{NR})}$. Here we have chosen suitable factors, $\lambda(T)=1 / T$, $1 / T^{2}, 1$, and $1 / T$, respectively, to make the spin chemical potential dimensionless. Note that Eq. (17) is the main assumption of this paper.

In the following, we use the $(3+1) \mathrm{D}$ hydrodynamic model CLVisc to calculate the four types of vorticities and then use Eq. (17) to obtain the spin vector and then the corresponding spin polarization.

\section{HYDRODYNAMIC MODEL}

The space-time evolution of the hot quark-gluon plasma and dense hadronic matter is described by second-order
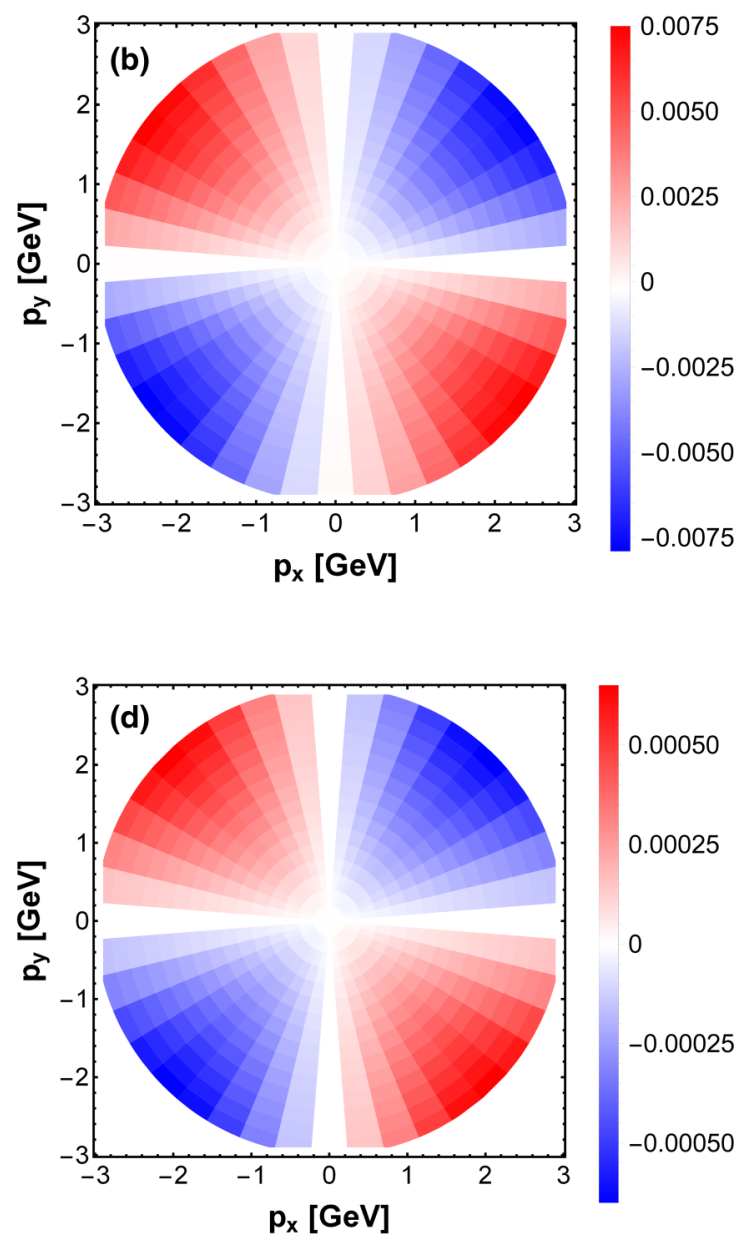

FIG. 2. The longitudinal polarization in $\mathrm{Au}+\mathrm{Au}$ collisions at $200 \mathrm{GeV}$ and $Y \in[-1,1]$ with the optical Glauber initial condition as functions of $\left(p_{x}, p_{y}\right)$. Panels (a)-(d) correspond to the thermal, kinematic, T, and NR vorticities, respectively. 
relativistic hydrodynamic equations,

$$
\nabla_{\mu} T^{\mu \nu}=0,
$$

where $T^{\mu \nu}=(\varepsilon+P) u^{\mu} u^{\nu}-P g^{\mu \nu}+\pi^{\mu \nu}$ is the energymomentum tensor which is symmetric, $\varepsilon$ is the local energy density in the comoving frame of the fluid, $P=$ $P(\varepsilon)$ is the pressure determined by the QCD equation of state, $u^{\mu}$ is the fluid four-velocity obeying $u_{\mu} u^{\mu}=1, g^{\mu \nu}=$ $\operatorname{diag}\left(1,-1,-1,-1 / \tau^{2}\right)$ is the metric tensor, and $\pi^{\mu \nu}$ is the shear-stress tensor whose evolution is solved using a separate group of equations,

$$
\pi^{\mu \nu}=\eta_{v} \sigma^{\mu \nu}-\tau_{\pi}\left[\triangle_{\alpha}^{\mu} \triangle_{\beta}^{v} u^{\lambda} \nabla_{\lambda} \pi^{\alpha \beta}+\frac{4}{3} \pi^{\mu \nu} \theta\right],
$$

where $\eta_{v}$ is the shear viscous coefficient; $\sigma^{\mu v} \equiv 2 \nabla^{\langle\mu} u^{v\rangle} \equiv$ $2 \triangle^{\mu \nu \alpha \beta} \nabla_{\alpha} u_{\beta}$ is the symmetric shear tensor; $\tau_{\pi}=5 \eta_{\nu} /(T s)$ is the relaxation time for the shear viscosity; $\triangle^{\mu \nu}=g^{\mu v}-u^{\mu} u^{v}$ is the projection operator that makes the resulting contracted vector orthogonal to $u^{\mu} ; \triangle^{\mu \nu \alpha \beta}=\frac{1}{2}\left(\triangle^{\mu \alpha} \triangle^{\nu \beta}+\triangle^{\mu \beta} \triangle^{\nu \alpha}\right)-$ $\frac{1}{3} \triangle^{\mu \nu} \triangle^{\alpha \beta}$ is the double projection operator that makes the resulting contracted tensor symmetric, traceless, and orthogonal to $u^{\mu}$; and $\theta \equiv \nabla_{\mu} u^{\mu}$ is the expansion rate. The operator $\nabla_{\mu}$ is the covariant derivative operator defined as

$$
\begin{gathered}
\nabla_{\mu} \lambda^{\nu}=\partial_{\mu} \lambda^{\nu}+\Gamma_{\mu \rho}^{\nu} \lambda^{\rho}, \\
\nabla_{\mu} \lambda^{\rho \sigma}=\partial_{\mu} \lambda^{\rho \sigma}+\Gamma_{\mu \lambda}^{\rho} \lambda^{\lambda \sigma}+\Gamma_{\mu \lambda}^{\sigma} \lambda^{\rho \lambda},
\end{gathered}
$$

for vectors $\lambda^{\mu}$ and tensors $\lambda^{\mu \nu}$. The $\Gamma^{\prime}$ s are Christoffel symbols solved as a function of $g^{\mu \nu}$ :

$$
\Gamma_{\rho \sigma}^{\mu}=\frac{1}{2} g^{\mu \lambda}\left(\partial_{\sigma} g_{\lambda \rho}+\partial_{\rho} g_{\lambda \sigma}-\partial_{\lambda} g_{\rho \sigma}\right) .
$$

The above $(3+1) \mathrm{D}$ viscous hydrodynamic equations are solved numerically using CLVisc $[77,78]$ with the s95p-PCE lattice QCD equation of state [88], and two different initial conditions, the optical Glauber initial condition without an initial OAM and the AMPT initial condition with an initial OAM, are applied to check the dependencies of the results on initial conditions.

\section{NUMERICAL RESULTS FOR HYPERON POLARIZATION}

In this section we present our numerical results for the polarization of $\Lambda$ hyperons through vorticity fields by Eq. (17). We choose the coordinate system for collisions of two gold nuclei at $200 \mathrm{GeV}$ in $20-50 \%$ centrality (see Fig. 1). The spatial indices $\mu=1,2$, and 3 in $S^{\mu}(p)$ in Eq. (17) correspond to the $x, y$, and $z$ directions, respectively, so sometimes we write $\mu=1,2$, and 3 as $\mu=x, y$, and $z$. To test the effect of different choices for the spin chemical potential coupled to the spin tensor, we choose four types of vorticities: the kinematic vorticity, the T vorticity, the thermal vorticity, and the NR vorticity. We use the hydrodynamic model CLVisc to compute the vorticity field on the freeze-out hypersurface.

We use two types of the initial condition: the optical Glauber initial condition without an initial OAM and the AMPT initial condition with an initial OAM. In the optical Glauber initial condition, the initial energy density distribution is boost invariant at midrapidity and is symmetric about the $y$ axis. As a result, it does not provide any initial OAM. On
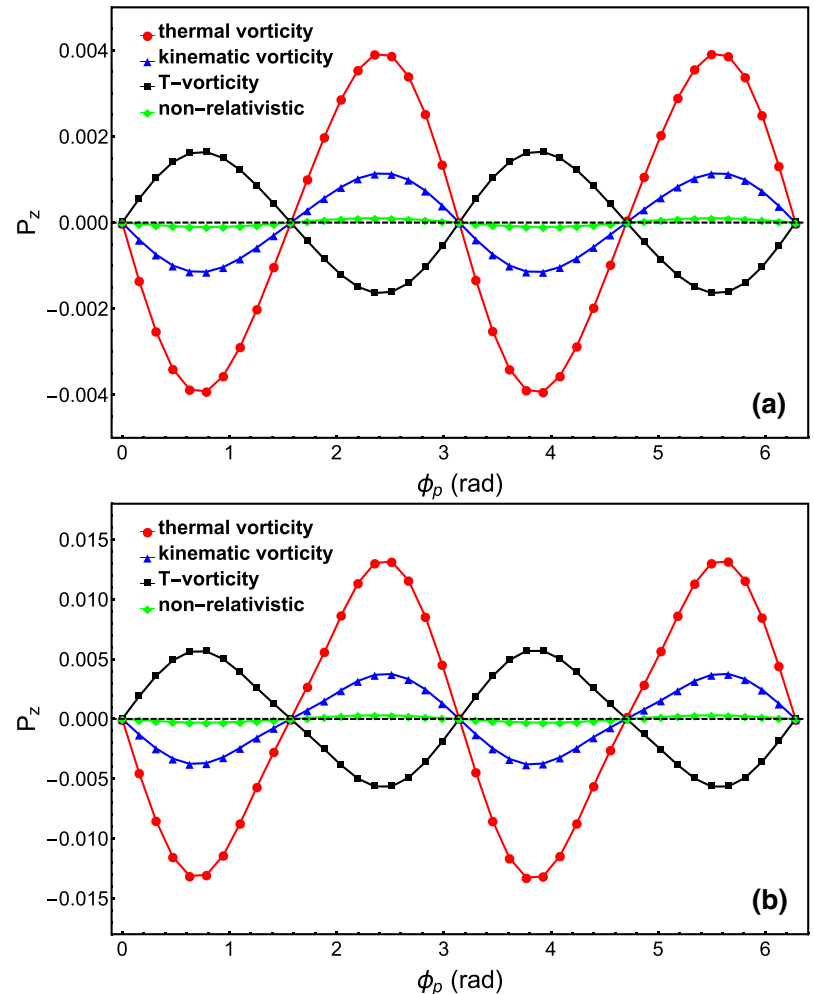

FIG. 3. The longitudinal polarizations as functions of azimuthal angles in transverse momenta in $\mathrm{Au}+\mathrm{Au}$ collisions with the optical Glauber initial condition. (a) $p_{T} \in[0,1.2] \mathrm{GeV}$. (b) $p_{T} \in[0,3] \mathrm{GeV}$.

the other hand, the AMPT initial condition uses HIJING strings. These strings are attached to forward and backward going participants whose distributions are not symmetric about the $y$ axis in noncentral collisions. On one side of the $y-z$ plane, the midpoints of those strings are shifted to forward rapidity in the projectile-going direction. On the other side of the $y-z$ plane, the midpoints of those strings are shifted to backward rapidity in the target-going direction. This forward-backward asymmetry in the AMPT model introduces a nonzero initial OAM along the negative $y$ axis. One should keep in mind that there is no forward-backward asymmetry if the length of the strings is infinity (before string fragmentation). In that case, all strings cover midrapidity. The system would be perfectly boost invariant along the space-time rapidity and symmetric about the $y$ axis. This corresponds to extreme high energy collisions where the initial OAM disappears at midrapidity. It is consistent with experimental observation that the global polarization is stronger in lower energy collisions. For collisions at low beam energies, the strings from the AMPT model have finite fluctuating lengths [89]. The lengths of strings are determined by the longitudinal-momentum differences between their two end points which are quarks and diquarks from the projectile and the target, whose longitudinal momenta are sampled from parton distribution functions. In this way, the lengths of the strings are finite and fluctuating. This helps to propagate the forward-backward asymmetry to left-right asymmetry at midrapidity, which is responsible for the initial OAM. 

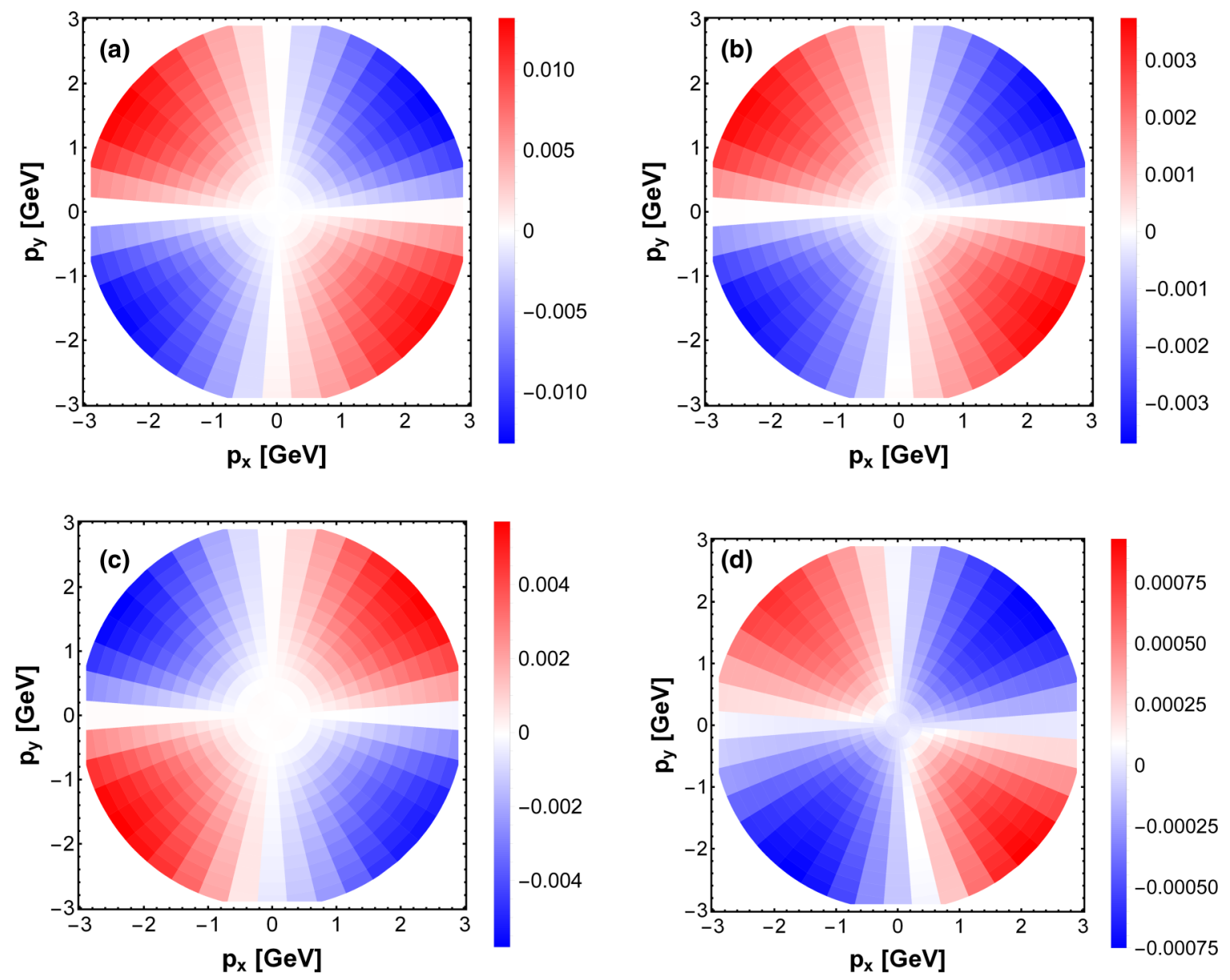

FIG. 4. The longitudinal polarizations in $\mathrm{Au}+\mathrm{Au}$ collisions at $200 \mathrm{GeV}$ in the rapidity range $Y \in[-1,1]$ with the AMPT initial condition as functions of $\left(p_{x}, p_{y}\right)$. Panels (a)-(d) correspond to the thermal, kinematic, T, and NR vorticities, respectively.

We calculate the transverse and longitudinal polarization of $\Lambda$ hyperons in the rapidity range $Y \in[-\Delta Y / 2, \Delta Y / 2]$ :

$$
\begin{aligned}
& \mathcal{P}_{x}(p)=\frac{2}{\Delta Y} \int_{-\Delta Y / 2}^{\Delta Y / 2} d Y S^{x}(p), \\
& \mathcal{P}_{y}(p)=\frac{2}{\Delta Y} \int_{-\Delta Y / 2}^{\Delta Y / 2} d Y S^{y}(p), \\
& \mathcal{P}_{z}(p)=\frac{2}{\Delta Y} \int_{-\Delta Y / 2}^{\Delta Y / 2} d Y S^{z}(p),
\end{aligned}
$$

where $S^{\mu}(p)$ is given by Eq. (17) and $p$ denotes the fourmomentum of the $\Lambda$ hyperon,

$$
p^{\mu}=\left(m_{T} \cosh Y, p_{x}, p_{y}, m_{T} \sinh Y\right),
$$

with $m_{T}=\sqrt{m_{\Lambda}^{2}+p_{x}^{2}+p_{y}^{2}}$. We choose the rapidity range $Y \in[-1,1]$ or $\Delta Y=2$ in the calculation.

\section{A. Results with optical Glauber initial condition}

With the optical Glauber initial condition, we present the results for the longitudinal polarization for $\Lambda$. Figure 2 shows $\mathcal{P}_{z}^{(i)}(p)$ for four types of spin chemical potentials or vorticities $i=K, T$, th, and NR. The transverse momentum $p_{x}$ and $p_{y}$ all range from -3 to $3 \mathrm{GeV}$. We see that the
$\mathrm{T}$ vorticity has the signs,,+-+ , and - from the first to the fourth quadrant consistent with the data. The kinematic, thermal, and NR vorticities have the signs,,-+- , and + , opposite in comparison with the data. Note that there is no contribution from the space-time gradient of the temperature in the kinematic vorticity; the temperature gradient part in the $\mathrm{T}$ vorticity has the sign opposite to that in the thermal vorticity. In Fig. 2 we see that the magnitude of $\mathcal{P}_{z}^{(K)}(p)$ is smaller than that of $\mathcal{P}_{z}^{(\text {th })}(p)$ and $\mathcal{P}_{z}^{(T)}(p)$, indicating the dominance of the temperature gradient parts in the thermal vorticity and the $\mathrm{T}$ vorticity.

The longitudinal polarizations from four types of vorticities as functions of azimuthal angles in transverse momenta are shown in Fig. 3. The azimuthal angle relative to the reaction plane $(z-x$ plane $)$ is defined as $\tan \phi_{p}=p_{y} / p_{x}$. The azimuthal angle distribution of the polarization $\overrightarrow{\mathcal{P}}\left(\phi_{p}\right)$ is obtained by taking an average over $p_{T}=\sqrt{p_{x}^{2}+p_{y}^{2}}$ for $\overrightarrow{\mathcal{P}}(p)$ :

$$
\overrightarrow{\mathcal{P}}\left(\phi_{p}\right)=\frac{1}{\Delta p_{T}} \int_{p_{T}^{\min }}^{p_{T}^{\max }} d p_{T} \overrightarrow{\mathcal{P}}(p),
$$

where $\Delta p_{T}=p_{T}^{\max }-p_{T}^{\min }$ denotes the range of the transverse momentum. In Fig. 3 we see that $\mathcal{P}_{z}^{(T)}\left(\phi_{p}\right) \sim \sin \left(2 \phi_{p}\right)$, which is consistent with the data, while all $\mathcal{P}_{z}^{(i)}\left(\phi_{p}\right) \sim-\sin \left(2 \phi_{p}\right)$ 

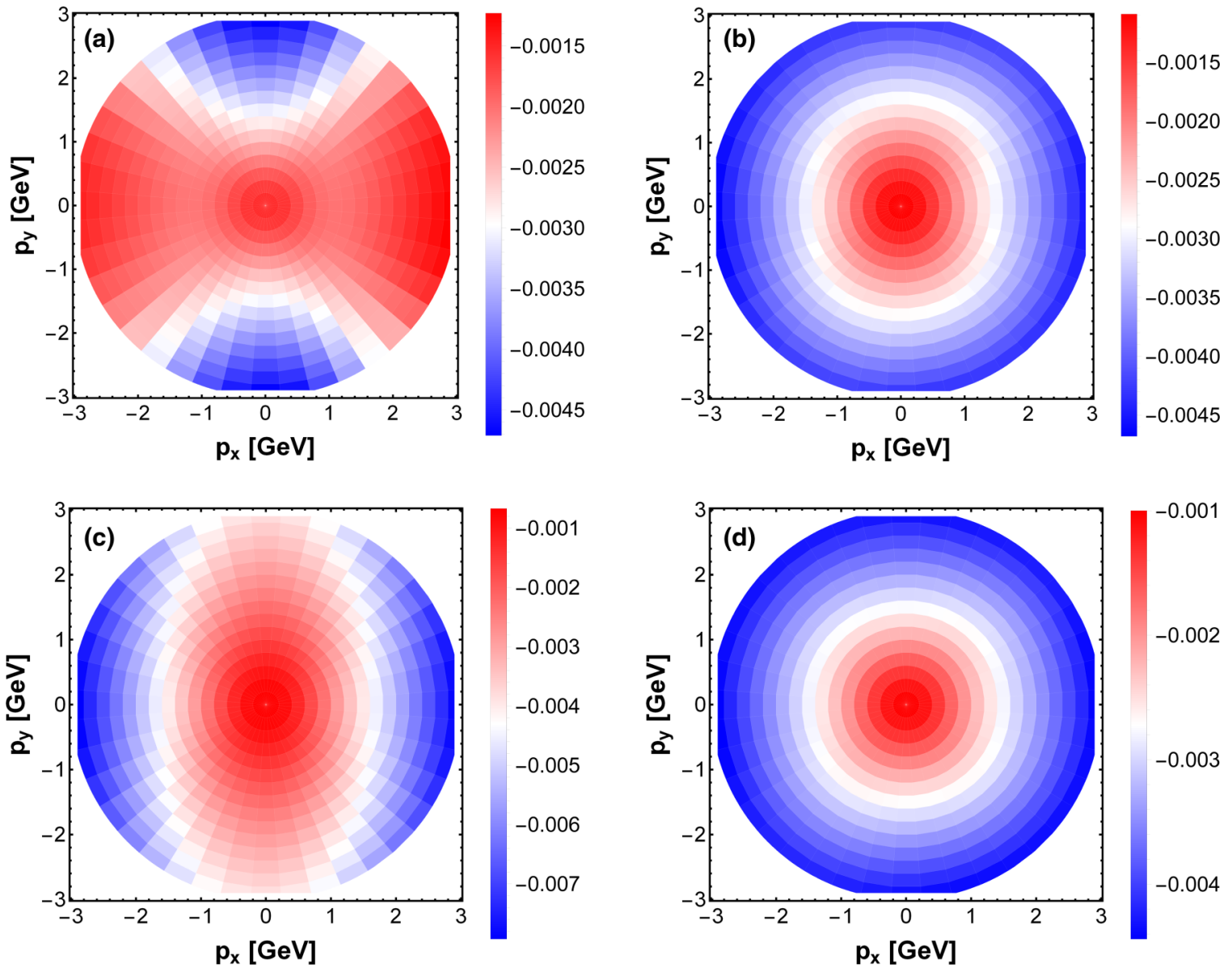

FIG. 5. The polarizations in the $-y$ direction. All other kinematic conditions are the same as those in Fig. 4.

with $i=K$, th, and NR, which have the wrong sign in comparison with the data. The magnitude of $\mathcal{P}_{z}^{\text {(th) }}\left(\phi_{p}\right)$ is the largest since it is the sum of the kinematic vorticity contribution and the temperature gradient contribution which have the same sign. However, in $\mathcal{P}_{z}^{(T)}\left(\phi_{p}\right)$ the kinematic vorticity and temperature gradient contribution have the opposite sign and the latter is dominant over the former. This is the reason that $\omega_{\mu \nu}^{(T)}(T)$ and $\omega_{\mu \nu}^{(\text {th) }}(T)$ have the opposite sign as shown in Eqs. (8) and (13).

Since there is no initial OAM in the optical Glauber initial condition, the polarizations in the $y$ direction, $\mathcal{P}_{y}(p)$, are vanishing for all four types of vorticities.

\section{B. Results with the AMPT initial condition}

In this subsection, we present the results for the AMPT initial condition which encodes the initial OAM of two nuclei in the $-y$ direction.

The results of $\mathcal{P}_{z}(p)$ and $\mathcal{P}_{y}(p)$ for four types of vorticities are shown in Figs. 4 and 5, respectively. We see in Fig. 4 that the signs of $\mathcal{P}_{z}(p)$ with the AMPT initial condition are the same as those with the Glauber initial condition, but the magnitudes of $\mathcal{P}_{z}(p)$ with the AMPT initial condition are smaller than those with the Glauber initial condition except $\mathcal{P}_{z}(p)$ for the NR vorticity that has almost the same magnitude with both initial conditions.
We can take an average over $p_{T}$ for $\mathcal{P}_{z}(p)$ in a transversemomentum range to obtain $\mathcal{P}_{z}\left(\phi_{p}\right)$. The results for $\mathcal{P}_{z}\left(\phi_{p}\right)$ are shown in Fig. 6 for two transverse-momentum ranges. We see that the magnitudes of $\mathcal{P}_{z}\left(\phi_{p}\right)$ become smaller in the $p_{T}$ range with smaller transverse momenta. For the range $p_{T} \in[0,1.2]$ $\mathrm{GeV}$, the magnitude of $\mathcal{P}_{z}\left(\phi_{p}\right)$ matches the data. But if we choose $p_{T} \in[0,3] \mathrm{GeV}$, the magnitude of $\mathcal{P}_{z}\left(\phi_{p}\right)$ is 1 order of magnitude larger than the data.

In contrast to the vanishing $\mathcal{P}_{y}(p)$ with the Glauber initial condition, we obtain finite values of $\mathcal{P}_{y}(p)$ in the AMPT initial condition as shown in Fig. 5. The results for $\mathcal{P}_{y}\left(\phi_{p}\right)$ are displayed in Fig. 7. All four types of vorticities give the correct sign of the initial OAM in the $-y$ direction. Note that only $\mathcal{P}_{y}^{(T)}\left(\phi_{p}\right)$ for the $\mathrm{T}$ vorticity gives the falling trend in $\phi_{p}$ consistent with the data. Although we have the correct trend in $\phi_{p}$ in $\mathcal{P}_{y}^{(T)}\left(\phi_{p}\right)$, our results fall slower than the data as $\phi_{p}$ increases. Our results for $\mathcal{P}_{y}^{(T)}\left(\phi_{p}\right)$ match the data at $\phi_{p}=0$, but at $\phi_{p}=\pi / 2$ our results are $\mathcal{P}_{y}^{(T)}\left(\phi_{p}\right) \approx 0.25$ while the data approach zero.

We also calculated $\mathcal{P}_{y}(Y)$ in $-y$ direction as functions of the rapidity $Y$, the results are shown in Fig. 8. We see that $\mathcal{P}_{y}(Y)$ is an even function of $Y$ and increases slowly with $|Y|$. The values of $\mathcal{P}_{y}(Y)$ are very close for the kinematic vorticity and the NR vorticity, and $\mathcal{P}_{y}(Y)$ for the $\mathrm{T}$ vorticity is the largest while that for thermal vorticity is the smallest. 


\section{With different average method in momentum}

We can also choose a different method of the average over transverse momenta and rapidity to replace Eqs. (23) and (25).
From Eq. (17) we can take an average of the denominator and numerator separately to obtain the $i$ th component of the polarization vector:

$$
\mathcal{P}_{i}\left(\phi_{p}\right)=-\frac{1}{4 m} \epsilon^{i \rho \sigma \tau} \frac{\int_{p_{T}^{\min }}^{p_{\max }^{\max }} d p_{T} p_{T} \int_{-\Delta Y / 2}^{\Delta Y / 2} d Y \int d \Sigma_{\lambda} p^{\lambda} p_{\tau} \Omega_{\rho \sigma} f_{\mathrm{FD}}\left(1-f_{\mathrm{FD}}\right)}{\int_{p_{T}^{\min }}^{p_{\max }^{\max }} d p_{T} p_{T} \int_{-\Delta Y / 2}^{\Delta Y / 2} d Y \int d \Sigma_{\lambda} p^{\lambda} f_{\mathrm{FD}}}+O\left(\Omega_{\mu \nu}^{2}\right) .
$$

Note that we have introduced an additional $p_{T}$ factor into the $p_{T}$ integrals in both the denominator and the numerator since it corresponds to the Lorentz invariant integral $d^{3} p / E_{p}$. The numerical results for $\mathcal{P}_{z}\left(\phi_{p}\right)$ are presented in Fig. 9. We see that, with the same cutoffs for $p_{T}$, the results for $\mathcal{P}_{z}\left(\phi_{p}\right)$ from Eq. (26) are a little larger than those from Eqs. (23) and (25). The same behavior also occurs in the results for $\mathcal{P}_{y}\left(\phi_{p}\right)$ with two different average methods.

\section{DISCUSSIONS}

We make some remarks about the results. We have checked the first two conditions in Eq. (15) and found that they are not fulfilled, so the use of the thermal vorticity as the spin chemical potential is not justified in the hydrosimulation.
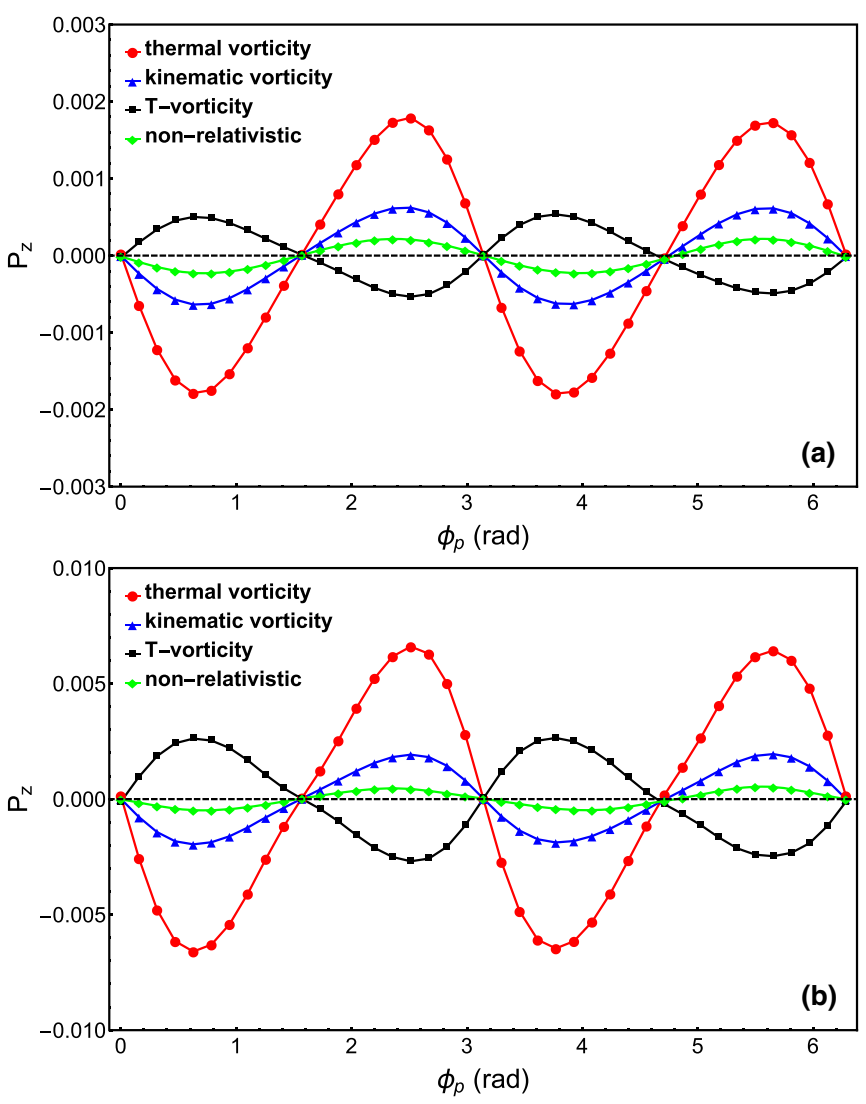

FIG. 6. The longitudinal polarizations as functions of azimuthal angles in transverse momenta in $\mathrm{Au}+\mathrm{Au}$ collisions with the AMPT initial condition. (a) $p_{T} \in[0,1.2] \mathrm{GeV}$. (b) $p_{T} \in[0,3] \mathrm{GeV}$.
For $\mathcal{P}_{y}\left(\phi_{p}\right)$, we find that only the $\mathrm{T}$ vorticity gives the right trend in $\phi_{p}$ in comparison to the data, although it decreases slower than the data. Except for the trend in $\phi_{p}$, all vorticities can give the global polarization consistent with the data. The reason why the $\mathrm{T}$ vorticity can give the right trend in $\phi_{p}$ may be understood as follows. The T vorticity is conserved so that the T-vorticity flux is frozen in the fluid and moves with the fluid cell. In this sense, we can regard the T-vorticity flux as a kind of conserved charge. At the early stage of a noncentral collision, the $\mathrm{T}$ vorticity in the out-of-plane direction may be induced by the global OAM; then as the pressure gradient is stronger in the in-plane direction than the out-of-plane direction, the $\mathrm{T}$ vorticity will have a positive elliptic flow which results in the unique $\phi_{p}$ dependence as shown in Fig. 7. This suggests that if the spin is (quasi-)conserved, after being polarized in the early stage by the OAM, the pressure gradient would lead to a $\phi_{p}$ dependence similar to that for the $\mathrm{T}$ vorticity. This may be verified by the simulation using spin hydrodynamics $[73,75]$.

We see very different and even opposite behaviors of $\mathcal{P}_{z}\left(\phi_{p}\right)$ from different vorticities. This might be related to the fact that $\mathcal{P}_{z}\left(\phi_{p}\right)$ is 1 order of magnitude smaller than $\mathcal{P}_{y}\left(\phi_{p}\right)$ since there is no initial OAM in the $z$ direction. Also $\mathcal{P}_{z}\left(\phi_{p}\right)$ is almost independent of $\mathcal{P}_{y}\left(\phi_{p}\right)$. This can be seen from the observation that the results of $\mathcal{P}_{z}\left(\phi_{p}\right)$ from all types of vorticities in the Glauber initial condition (without an initial OAM) have the same behaviors as in the AMPT initial condition (with an initial OAM). In the optical Glauber initial condition, we found that $\mathcal{P}_{y}\left(\phi_{p}\right)$ from all types of vorticities

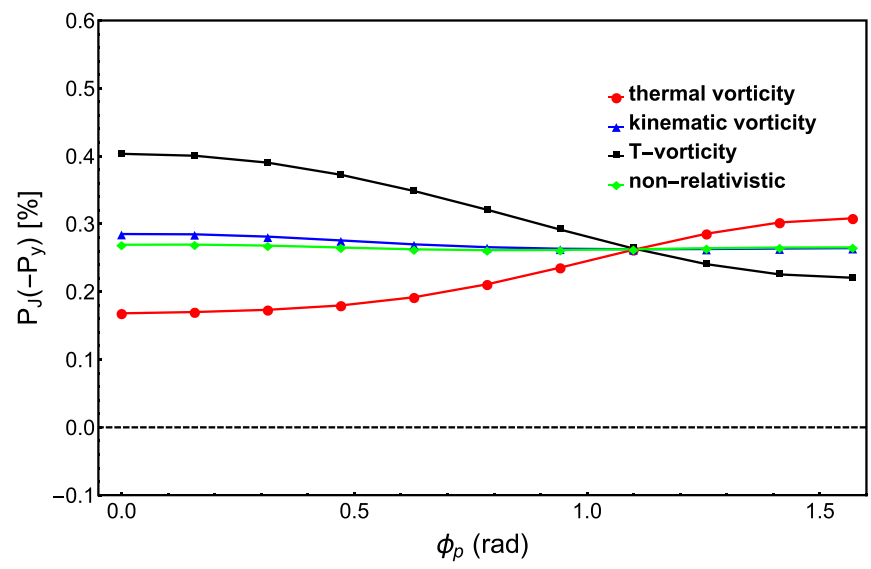

FIG. 7. The polarizations in the $-y$ direction as functions of azimuthal angles in transverse momenta in $\mathrm{Au}+\mathrm{Au}$ collisions with the AMPT initial condition. The transverse-momentum range is set to $p_{T} \in[0,3] \mathrm{GeV}$. 


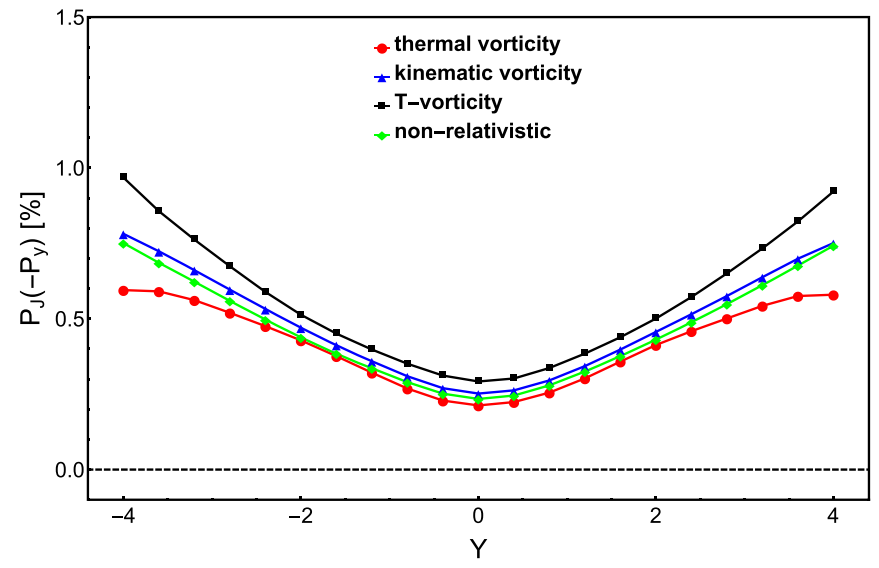

FIG. 8. The polarizations in the $-y$ direction as functions of the rapidity in $\mathrm{Au}+\mathrm{Au}$ collisions with the AMPT initial condition. The transverse-momentum range is set to $p_{T} \in[0,3] \mathrm{GeV}$.

are vanishing since there is no orbital angular momentum encoded in the initial state.

Only the $\mathrm{T}$ vorticity in our simulation can describe the data of $\mathcal{P}_{z}\left(\phi_{p}\right)$ which is the main finding of the paper. The temperature part $\omega_{\mu \nu}^{(T)}(T)$ in the T vorticity (8) plays an essential role in producing the right sign of $\mathcal{P}_{z}\left(\phi_{p}\right)$ : the sign of $\omega_{\mu \nu}^{(T)}(T)$ is different from that of $\omega_{\mu \nu}^{(K)}$ but with larger magnitude, so the T vorticity takes the sign of $\omega_{\mu \nu}^{(T)}(T)$. It is just the opposite way for the thermal vorticity (13) to make its sign: the temperature part $\omega_{\mu \nu}^{(\text {th })}(T)$ has the same sign as $\omega_{\mu \nu}^{(K)}$.

The implication of the $\mathrm{T}$ vorticity by the data may possibly indicate the following.

(i) The time behavior of the temperature at the freeze-out is essential for the $\mathrm{T}$ vorticity to reproduce the correct sign of $\mathcal{P}_{z}\left(\phi_{p}\right)$.

(ii) The $\mathrm{T}$ vorticity might be coupled with the spin in a way similar to that in which a magnetic moment is coupled to a magnetic field. Considering an ideal fluid without a conserved charge density (such as the baryon number density) which is

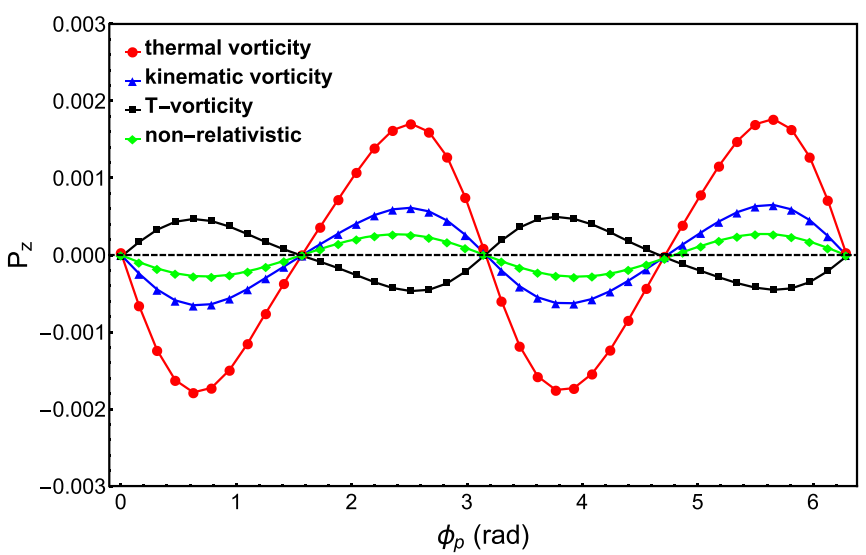

FIG. 9. The longitudinal polarizations as functions of azimuthal angles in transverse momenta in $\mathrm{Au}+\mathrm{Au}$ collisions with the AMPT initial condition. An alternative average method corresponding to Eq. (26) is used. The $p_{T}$ range is chosen to be $p_{T} \in[0,1.2] \mathrm{GeV}$ to match the magnitude of the data. the case in the current hydrosimulation for high energy heavy ion collisions, $T u^{\mu}$ can be regarded as a vector potential and the T-vorticity tensor is then the corresponding field-strength tensor, so the conservation of T-vorticity flux is similar to the conservation of the magnetic flux in an ideally conducting fluid [see Eqs. (11) and (12)]. However, such a picture is not yet rigorously established and it is also unclear how the roles of the $\mathrm{T}$ vorticity and the thermal vorticity change when the system approaches global equilibrium. Nevertheless, for collisions at lower energies in which the baryon number density is finite, the conservation of the T-vorticity flux does not hold anymore [85]. Thus, the behavior of $\mathcal{P}_{z}\left(\phi_{p}\right)$ in low energy collisions might provide a test of this point of view.

(iii) The assumption that the spin chemical potential can be constructed using $T$ and $u^{\mu}$ might not be correct, so the fact that the $T$ vorticity can qualitatively reproduce the experimental data for $\mathcal{P}_{z}\left(\phi_{p}\right)$ and $\mathcal{P}_{y}\left(\phi_{p}\right)$ is just accidental. This may be tested by using the spin hydrodynamics, which is, however, beyond the scope of this work and we leave it for the future.

(iv) It is also possible that it is a coincidence from the main assumption that the spin vector is given by the $\mathrm{T}$ vorticity in the same way as the thermal vorticity.

The true relationship between the spin vector on the freezeout hypersurface and all these vorticities is unclear and has to be figured out.

All our results depend on a set of parameters and assumptions. For $\mathcal{P}_{z}\left(\phi_{p}\right)$ and $\mathcal{P}_{y}\left(\phi_{p}\right)$, one of the most sensitive parameters is the cutoffs in $p_{T}$ in Eq. (25). For example, as shown in Fig. 6, if we choose the range $p_{T} \in[0,1.2] \mathrm{GeV}$, the theoretical results match the data of $\mathcal{P}_{z}\left(\phi_{p}\right)$. However, if we choose the larger range $p_{T} \in[0,3] \mathrm{GeV}$, our theoretical results are much larger than the data of $\mathcal{P}_{z}\left(\phi_{p}\right)$. The aim of this paper is a qualitative study instead of a quantitative one. We will carry out a detailed and quantitative study of the effects in the future.

\section{SUMMARY}

There is a disagreement between theoretical model calculations and recent experimental data about the azimuthal angle dependence of both the longitudinal and transverse polarization of hyperons. These theoretical models are mainly based on the hydrodynamic or kinetic descriptions of the fluid vorticity and express the spin polarization in terms of the thermal vorticity. However, away from global equilibrium, the linear relationship between the spin polarization and thermal vorticity may not be valid (higher order contribution might be relevant). Instead, the spin polarization (or equivalently the spin chemical potential) itself should be regarded as a dynamical variable. Recently there have been attempts in formulating the theory of relativistic hydrodynamics with the spin chemical potential as a (quasi-)hydrodynamic variable, but so far there has been no reliable numerical implementation of the spin hydrodynamics in the market yet.

In this paper, we assume that the spin vector is determined from the spin chemical potential $\Omega_{\mu \nu}$ in the same way as from the thermal vorticity when the thermal vorticity is small [see Eqs. (16) and (17)]. We also assume that the spin chemical potential $\Omega_{\mu \nu}$ is still determined by the fluid velocity and 
temperature, which means that $\Omega_{\mu \nu}$ can be regarded as being proportional to a type of vorticity. In relativistic hydrodynamics there are various types of vorticities such as the kinematic, temperature, and thermal vorticities. There is also a relativistic extension of the nonrelativistic vorticity. We thus explore the possibility that the spin chemical potential is proportional to these four vorticities and the spin vector is given by Eq. (17).

We use CLVisc, a $(3+1)$ D viscous hydrodynamic model, to compute the vorticity field. We choose two different initial conditions for the hydrosimulation: the optical Glauber one without an initial orbital angular momentum and the AMPT one with an initial orbital angular momentum. We calculated $\mathcal{P}_{z}\left(\phi_{p}\right)$ and $\mathcal{P}_{y}\left(\phi_{p}\right)$ as functions of $\phi_{p}$, the azimuthal angle in transverse momentum, for four types of vorticities: the kinematic, temperature, thermal, and relativistic extension of the nonrelativistic vorticity. Our results showthe following.

(i) All types of vorticities have the correct sign of $\mathcal{P}_{y}$ for the AMPT initial condition. With the optical Glauber initial condition, they all give vanishing results for $\mathcal{P}_{y}\left(\phi_{p}\right)$ since there is no orbital angular momentum encoded in the initial state. For $\mathcal{P}_{y}\left(\phi_{p}\right)$ with the AMPT initial condition, only the temperature vorticity has the same trend as the data, although its magnitude does not agree with the data.

(ii) For the azimuthal angle distribution in the longitudinal polarization, $\mathcal{P}_{z}\left(\phi_{p}\right)$, only the temperature vorticity reproduces the sign of the oscillation in the azimuthal angle in the data. The other three types of vorticities have a difference in sign from the data.

(iii) The oscillation behavior of $\mathcal{P}_{z}\left(\phi_{p}\right)$ (not the magnitude) is insensitive to the initial conditions with or without the orbital angular momentum being encoded.

\section{ACKNOWLEDGMENTS}

The authors thank F. Becattini, X.-L. Sheng, and X.-L. Xia for insightful discussions. H.-Z.W. and Q.W. are supported in part by the National Natural Science Foundation of China (NSFC) under Grants No. 11535012 and No. 11890713 and the Key Research Program of the Chinese Academy of Sciences under Grant No. XDPB09. X.-G.H. is supported by the NSFC under Grants No. 11535012 and No. 11675041.
[1] A. Einstein and W. J. de Haas, Experimenteller nachweis der ampereschen molekularströme, Dtsch. Phys. Ges., Verh. 17, 152 (1915).

[2] S. J. Barnett, Gyromagnetic and electron-inertia effects, Rev. Mod. Phys. 7, 129 (1935).

[3] R. Takahashi, M. Matsuo, M. Ono, K. Harii, H. Chudo, S. Okayasu, J. Ieda, S. Takahashi, S. Maekawa, and E. Saitoh, Spin hydrodynamic generation, Nat. Phys. 12, 52 (2016).

[4] Z.-T. Liang and X.-N. Wang, Globally Polarized Quark-Gluon Plasma in Noncentral $A+A$ Collisions, Phys. Rev. Lett. 94, 102301 (2005); 96, 039901(E) (2006).

[5] F. Becattini, F. Piccinini, and J. Rizzo, Angular momentum conservation in heavy ion collisions at very high energy, Phys. Rev. C 77, 024906 (2008).

[6] J.-H. Gao, S.-W. Chen, W.-T. Deng, Z.-T. Liang, Q. Wang, and X.-N. Wang, Global quark polarization in noncentral $A+A$ collisions, Phys. Rev. C 77, 044902 (2008).

[7] X.-G. Huang, P. Huovinen, and X.-N. Wang, Quark polarization in a viscous quark-gluon plasma, Phys. Rev. C 84, 054910 (2011).

[8] Q. Wang, Global and local spin polarization in heavy ion collisions: A brief overview, in Proceedings of the 26th International Conference on Ultra-relativistic Nucleus-Nucleus Collisions (Quark Matter 2017): Chicago, Illinois, USA, February 5-11, 2017, Nucl. Phys. A 967, 225 (2017).

[9] F. Becattini, G. Inghirami, V. Rolando, A. Beraudo, L. Del Zanna, A. De Pace, M. Nardi, G. Pagliara, and V. Chandra, A study of vorticity formation in high energy nuclear collisions, Eur. Phys. J. C 75, 406 (2015); 78, 354(E) (2018).

[10] L.-G. Pang, H. Petersen, Q. Wang, and X.-N. Wang, Vortical Fluid and $\Lambda$ Spin Correlations in High-Energy Heavy-Ion Collisions, Phys. Rev. Lett. 117, 192301 (2016).

[11] W.-T. Deng and X.-G. Huang, Vorticity in heavy-ion collisions, Phys. Rev. C 93, 064907 (2016).

[12] Y. Jiang, Z.-W. Lin, and J. Liao, Rotating quark-gluon plasma in relativistic heavy ion collisions, Phys. Rev. C 94, 044910 (2016); 95, 049904(E) (2017).
[13] F. Becattini, V. Chandra, L. Del Zanna, and E. Grossi, Relativistic distribution function for particles with spin at local thermodynamical equilibrium, Ann. Phys. 338, 32 (2013).

[14] R.-H. Fang, L.-G. Pang, Q. Wang, and X.-N. Wang, Polarization of massive fermions in a vortical fluid, Phys. Rev. C 94, 024904 (2016).

[15] Z.-T. Liang and X.-N. Wang, Spin alignment of vector mesons in non-central A + A collisions, Phys. Lett. B 629, 20 (2005).

[16] S. A. Voloshin, Polarized secondary particles in unpolarized high energy hadron-hadron collisions? arXiv:nucl-th/0410089.

[17] B. Betz, M. Gyulassy, and G. Torrieri, Polarization probes of vorticity in heavy ion collisions, Phys. Rev. C 76, 044901 (2007).

[18] L. Adamczyk et al. (STAR Collaboration), Global $\Lambda$ hyperon polarization in nuclear collisions: Evidence for the most vortical fluid, Nature (London) 548, 62 (2017)

[19] J. Adam et al. (STAR Collaboration), Global polarization of $\Lambda$ hyperons in $\mathrm{Au}+\mathrm{Au}$ collisions at $\sqrt{s_{N N}}=200 \mathrm{GeV}$, Phys. Rev. C 98, 014910 (2018).

[20] F. Becattini and E. Grossi, Quantum corrections to the stressenergy tensor in thermodynamic equilibrium with acceleration, Phys. Rev. D 92, 045037 (2015).

[21] F. Becattini, I. Karpenko, M. A. Lisa, I. Upsal, and S. A. Voloshin, Global hyperon polarization at local thermodynamic equilibrium with vorticity, magnetic field, and feed-down, Phys. Rev. C 95, 054902 (2017).

[22] W. Florkowski, B. Friman, A. Jaiswal, R. Ryblewski, and E. Speranza, Spin-dependent distribution functions for relativistic hydrodynamics of spin-1/2 particles, Phys. Rev. D 97, 116017 (2018).

[23] W. Florkowski, A. Kumar, and R. Ryblewski, Thermodynamic versus kinetic approach to polarization-vorticity coupling, Phys. Rev. C 98, 044906 (2018).

[24] U. W. Heinz, Kinetic Theory for Plasmas with Non-Abelian Interactions, Phys. Rev. Lett. 51, 351 (1983).

[25] H.-T. Elze, M. Gyulassy, and D. Vasak, Transport equations for the QCD quark Wigner operator, Nucl. Phys. B 276, 706 (1986). 
[26] D. Vasak, M. Gyulassy, and H.-T. Elze, Quantum transport theory for Abelian plasmas, Ann. Phys. 173, 462 (1987).

[27] P. Zhuang and U. W. Heinz, Relativistic quantum transport theory for electrodynamics, Ann. Phys. 245, 311 (1996).

[28] W. Florkowski, J. Hufner, S. P. Klevansky, and L. Neise, Chirally invariant transport equations for quark matter, Ann. Phys. 245, 445 (1996).

[29] J.-P. Blaizot and E. Iancu, The quark gluon plasma: Collective dynamics and hard thermal loops, Phys. Rep. 359, 355 (2002).

[30] Q. Wang, K. Redlich, H. Stöcker, and W. Greiner, Kinetic Equation for Gluons in the Background Gauge of QCD, Phys. Rev. Lett. 88, 132303 (2002).

[31] J.-H. Gao, Z.-T. Liang, S. Pu, Q. Wang, and X.-N. Wang, Chiral Anomaly and Local Polarization Effect from Quantum Kinetic Approach, Phys. Rev. Lett. 109, 232301 (2012).

[32] J.-W. Chen, S. Pu, Q. Wang, and X.-N. Wang, Berry Curvature and Four-Dimensional Monopoles in the Relativistic Chiral Kinetic Equation, Phys. Rev. Lett. 110, 262301 (2013).

[33] J.-H. Gao and Q. Wang, Magnetic moment, vorticity-spin coupling and parity-odd conductivity of chiral fermions in 4-dimensional Wigner functions, Phys. Lett. B 749, 542 (2015).

[34] Y. Hidaka, S. Pu, and D.-L. Yang, Relativistic chiral kinetic theory from quantum field theories, Phys. Rev. D 95, 091901(R) (2017).

[35] J.-H. Gao, S. Pu, and Q. Wang, Covariant chiral kinetic equation in the Wigner function approach, Phys. Rev. D 96, 016002 (2017).

[36] J.-H. Gao, Z.-T. Liang, Q. Wang, and X.-N. Wang, Disentangling covariant Wigner functions for chiral fermions, Phys. Rev. D 98, 036019 (2018).

[37] A. Huang, S. Shi, Y. Jiang, J. Liao, and P. Zhuang, Complete and consistent chiral transport from Wigner function formalism, Phys. Rev. D 98, 036010 (2018).

[38] J.-H. Gao, J.-Y. Pang, and Q. Wang, The chiral vortical effect in Wigner function approach, Phys. Rev. D 100, 016008 (2019).

[39] Y.-C. Liu, L.-L. Gao, K. Mameda, and X.-G. Huang, Chiral kinetic theory in curved spacetime, Phys. Rev. D 99, 085014 (2019).

[40] A. Vilenkin, Equilibrium partity-violating current in a magnetic field, Phys. Rev. D 22, 3080 (1980).

[41] D. E. Kharzeev, L. D. McLerran, and H. J. Warringa, The effects of topological charge change in heavy ion collisions: "Event by event P and CP violation," Nucl. Phys. A 803, 227 (2008).

[42] K. Fukushima, D. E. Kharzeev, and H. J. Warringa, The chiral magnetic effect, Phys. Rev. D 78, 074033 (2008).

[43] D. Kharzeev, K. Landsteiner, A. Schmitt, and H.-U. Yee (eds.), Strongly Interacting Matter in Magnetic Fields, Lecture Notes in Physics Vol. 871 (Springer, Berlin, 2013).

[44] D. E. Kharzeev, J. Liao, S. A. Voloshin, and G. Wang, Chiral magnetic and vortical effects in high-energy nuclear collisions-A status report, Prog. Part. Nucl. Phys. 88, 1 (2016).

[45] X.-G. Huang, Electromagnetic fields and anomalous transports in heavy-ion collisions-A pedagogical review, Rep. Prog. Phys. 79, 076302 (2016).

[46] K. Hattori and X.-G. Huang, Novel quantum phenomena induced by strong magnetic fields in heavy-ion collisions, Nucl. Sci. Tech. 28, 26 (2017).

[47] A. Vilenkin, Parity violating currents in thermal radiation, Phys. Lett. B 80, 150 (1978).
[48] J. Erdmenger, M. Haack, M. Kaminski, and A. Yarom, Fluid dynamics of R-charged black holes, J. High Energy Phys. 01 (2009) 055.

[49] N. Banerjee, J. Bhattacharya, S. Bhattacharyya, S. Dutta, R. Loganayagam, and P. Surowka, Hydrodynamics from charged black branes, J. High Energy Phys. 01 (2011) 094.

[50] D. T. Son and P. Surowka, Hydrodynamics with Triangle Anomalies, Phys. Rev. Lett. 103, 191601 (2009).

[51] D.-F. Hou, H. Liu, and H.-C. Ren, A possible higher order correction to the vortical conductivity in a gauge field plasma, Phys. Rev. D 86, 121703(R) (2012).

[52] N. Weickgenannt, X.-L. Sheng, E. Speranza, Q. Wang, and D. H. Rischke, Kinetic theory for massive spin-1/2 particles from the Wigner-function formalism, Phys. Rev. D 100, 056018 (2019).

[53] J.-H. Gao and Z.-T. Liang, Relativistic quantum kinetic theory for massive fermions and spin effects, Phys. Rev. D 100, 056021 (2019).

[54] K. Hattori, Y. Hidaka, and D.-L. Yang, Axial kinetic theory for massive fermions, arXiv:1903.01653.

[55] Z. Wang, X. Guo, S. Shi, and P. Zhuang, Mass correction to chiral kinetic equations, Phys. Rev. D 100, 014015 (2019).

[56] M. Baznat, K. Gudima, A. Sorin, and O. Teryaev, Helicity separation in heavy-ion collisions, Phys. Rev. C 88, 061901(R) (2013).

[57] L. P. Csernai, V. K. Magas, and D. J. Wang, Flow vorticity in peripheral high-energy heavy-ion collisions, Phys. Rev. C 87, 034906 (2013).

[58] L. P. Csernai, D. J. Wang, M. Bleicher, and H. Stocker, Vorticity in peripheral collisions at the Facility for Antiproton and Ion Research and at the JINR Nuclotron-based Ion Collider fAcility, Phys. Rev. C 90, 021904(R) (2014).

[59] O. Teryaev and R. Usubov, Vorticity and hydrodynamic helicity in heavy-ion collisions in the hadron-string dynamics model, Phys. Rev. C 92, 014906 (2015).

[60] Yu. B. Ivanov and A. A. Soldatov, Vorticity in heavy-ion collisions at the JINR Nuclotron-based Ion Collider fAcility, Phys. Rev. C 95, 054915 (2017).

[61] H. Li, L.-G. Pang, Q. Wang, and X.-L. Xia, Global $\Lambda$ polarization in heavy-ion collisions from a transport model, Phys. Rev. C 96, 054908 (2017).

[62] D.-X. Wei, W.-T. Deng, and X.-G. Huang, Thermal vorticity and spin polarization in heavy-ion collisions, Phys. Rev. C 99 014905 (2019).

[63] I. Karpenko and F. Becattini, Study of $\Lambda$ polarization in relativistic nuclear collisions at $\sqrt{s_{N N}}=7.7-200 \mathrm{GeV}$, Eur. Phys. J. 77, 213 (2017).

[64] Y. Xie, D. Wang, and L. P. Csernai, Global $\Lambda$ polarization in high energy collisions, Phys. Rev. C 95, 031901(R) (2017).

[65] Y. Sun and C. M. Ko, $\Lambda$ hyperon polarization in relativistic heavy ion collisions from a chiral kinetic approach, Phys. Rev. C 96, 024906 (2017).

[66] J. Adam et al. (STAR Collaboration), Polarization of $\Lambda(\bar{\Lambda})$ Hyperons along the Beam Direction in $\mathrm{Au}+\mathrm{Au}$ Collisions at $\sqrt{s_{N N}}=200 \mathrm{GeV}$, Phys. Rev. Lett. 123, 132301 (2019).

[67] F. Becattini and Iu. Karpenko, Collective Longitudinal Polarization in Relativistic Heavy-Ion Collisions at Very High Energy, Phys. Rev. Lett. 120, 012302 (2018). 
[68] X.-L. Xia, H. Li, Z.-B. Tang, and Q. Wang, Probing vorticity structure in heavy-ion collisions by local $\Lambda$ polarization, Phys. Rev. C 98, 024905 (2018).

[69] Y. Sun and C. M. Ko, Azimuthal angle dependence of the longitudinal spin polarization in relativistic heavy ion collisions, Phys. Rev. C 99, 011903(R) (2019).

[70] X.-L. Xia, H. Li, X.-G. Huang, and H. Z. Huang, Feed-down effect on $\Lambda$ spin polarization, Phys. Rev. C 100, 014913 (2019).

[71] F. Becattini, G. Cao, and E. Speranza, Polarization transfer in hyperon decays and its effect in relativistic nuclear collisions, Eur. Phys. J C 79, 741 (2019).

[72] F. Becattini, W. Florkowski, and E. Speranza, Spin tensor and its role in non-equilibrium thermodynamics, Phys. Lett. B 789, 419 (2019).

[73] W. Florkowski, B. Friman, A. Jaiswal, and E. Speranza, Relativistic fluid dynamics with spin, Phys. Rev. C 97, 041901(R) (2018).

[74] W. Florkowski and R. Ryblewski, Hydrodynamics with spinPseudo-gauge transformations, semi-classical expansion, and Pauli-Lubanski vector, Prog. Part. Nucl. Phys. 108, 103709 (2019).

[75] K. Hattori, M. Hongo, X.-G. Huang, M. Matsuo, and H. Taya, Fate of spin polarization in a relativistic fluid: An entropycurrent analysis, Phys. Lett. B 795, 100 (2019).

[76] J.-J. Zhang, R.-H. Fang, Q. Wang, and X.-N. Wang, A microscopic description for polarization in particle scatterings, arXiv:1904.09152.

[77] L. Pang, Q. Wang, and X.-N. Wang, Effects of initial flow velocity fluctuation in event-by-event $(3+1) \mathrm{D}$ hydrodynamics, Phys. Rev. C 86, 024911 (2012).

[78] L.-G. Pang, H. Petersen, and X.-N. Wang, Pseudorapidity distribution and decorrelation of anisotropic flow within the opencomputing-language implementation CLVisc hydrodynamics, Phys. Rev. C 97, 064918 (2018).
[79] D. N. Zubarev, A. V. Prozorkevich, and S. A. Smolyanskii, Derivation of nonlinear generalized equations of quantum relativistic hydrodynamics, Teor. Mat. Fiz. 40, 821 (1979).

[80] Ch. G. van Weert, Maximum entropy principle and relativistic hydrodynamics, Ann. Phys. 140, 133 (1982).

[81] F. Becattini, L. Bucciantini, E. Grossi, and L. Tinti, Local thermodynamical equilibrium and the $\beta$ frame for a quantum relativistic fluid, Eur. Phys. J. C 75, 191 (2015).

[82] T. Hayata, Y. Hidaka, T. Noumi, and M. Hongo, Relativistic hydrodynamics from quantum field theory on the basis of the generalized Gibbs ensemble method, Phys. Rev. D 92, 065008 (2015).

[83] I. Karpenko and F. Becattini, Lambda polarization in heavy ion collisions: From RHIC BES to LHC energies, in Proceedings of the 27th International Conference on Ultrarelativistic NucleusNucleus Collisions (Quark Matter 2018): Venice, Italy, May 14-19, 2018, Nucl. Phys. A 982, 519 (2019).

[84] W. Florkowski, A. Kumar, and R. Ryblewski, Longitudinal spin polarization in a thermal model, arXiv:1904.00002.

[85] J.-H. Gao, B. Qi, and S.-Y. Wang, Vorticity and magnetic field production in relativistic ideal fluids, Phys. Rev. D 90, 083001 (2014).

[86] Y.-C. Liu, K. Mameda, and X.-G. Huang, Spin polarization of Dirac fermions in quantum field theory (unpublished).

[87] F. Becattini, Covariant Statistical Mechanics and the StressEnergy Tensor, Phys. Rev. Lett. 108, 244502 (2012).

[88] Sz. Borsanyi, G. Endrodi, Z. Fodor, S. D. Katz, and K. K. Szabo, Precision SU(3) lattice thermodynamics for a large temperature range, J. High Energy Phys. 07 (2012) 056.

[89] L.-G. Pang, H. Petersen, G.-Y. Qin, V. Roy, and X.-N. Wang, Decorrelation of anisotropic flow along the longitudinal direction, Eur. Phys. J. A 52, 97 (2016). 